\title{
Promotion of exclusive breast-feeding at scale within routine health services: impact of breast-feeding counselling training for community health workers in Recife, Brazil
}

\author{
Sonia B Coutinho' ${ }^{1}$, Pedro IC Lira ${ }^{2}$, Marilia C Lima' ${ }^{1}$, Paulo G Frias ${ }^{3}$, \\ Sophie H Eickmann ${ }^{1}$ and Ann Ashworth,* \\ 'Department of Maternal and Child Health, Federal University of Pernambuco, Recife, Brazil: ${ }^{2}$ Department of \\ Nutrition, Federal University of Pernambuco, Recife, Brazil: ${ }^{3}$ Municipal Health Secretariat, Recife, Brazil: \\ ${ }^{4}$ Department of Population Health, London School of Hygiene \& Tropical Medicine, Keppel Street, London \\ WCIE 7HT, UK
}

Submitted 9 September 2012: Final revision received 9 May 2013: Accepted 29 May 2013: First published online 11 July 2013

\begin{abstract}
Objective: Breast-feeding counselling has been identified as the intervention with the greatest potential for reducing child deaths, but there is little experience in delivering breast-feeding counselling at scale within routine health systems. The study aim was to compare rates of exclusive breast-feeding associated with a breast-feeding counselling intervention in which community health agents (CHA) received $20 \mathrm{~h}$ of training directed at counselling and practical skills with rates pre-intervention when $\mathrm{CHA}$ received $4 \mathrm{~h}$ of didactic teaching.

Design: Cross-sectional surveys of breast-feeding practices were conducted pre- and post-intervention in random samples of 1266 and 1245 infants aged 0-5.9 months, respectively.

Setting: Recife, Brazil, with a population of 2 million.

Subjects: CHA ( $n$ 1449) of Brazil's Family Health Programme were trained to provide breast-feeding counselling at home visits.

Results: Rates of exclusive breast-feeding improved when CHA were trained to provide breast-feeding counselling and were significantly higher by 10-13 percentage points at age $3-5.9$ months when compared with pre-intervention rates $(P<0 \cdot 05)$. Post-intervention point prevalence of exclusive breast-feeding for infants aged $<4$ months was $63 \%$ and for those aged $<6$ months was $50 \%$. Conclusions: Multifunctional CHA were able to deliver breast-feeding counselling at scale within a routine health service and this was associated with a significant increase in rates of exclusive breast-feeding. The study reinforces the need to focus training on counselling and practical skills; a key component was an interactive style that utilized the knowledge and experience of CHA. The findings are relevant to the call by international organizations to scale up breast-feeding counselling.
\end{abstract}

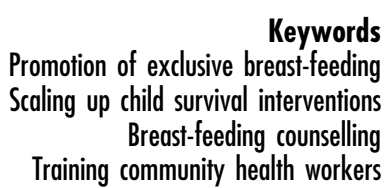

Child mortality is unacceptably high in low-income countries and in poor areas of middle-income countries, and counselling about breast-feeding has been identified as the intervention with the greatest potential for reducing child deaths ${ }^{(1,2)}$. Consequently there has been a call for breast-feeding counselling interventions to be rapidly implemented at scale.

The three main strategies for delivering breast-feeding counselling are the Baby Friendly Hospital Initiative (BFHI), peer counsellors and community health workers. The BFHI is the most widely used system and can achieve high rates of exclusive breast-feeding in hospital, but high rates are not generally sustained once mothers return home ${ }^{(3-5)}$. In contrast, trials of lay or peer counsellors have found substantial increases in rates of exclusive breast-feeding during the first 6 months $^{(6-14)}$ but the sustainability of systems dependent on unpaid counsellors has been questioned ${ }^{(15)}$. Programmes that are integrated into routine health services may prove more enduring ${ }^{(16)}$ and in India multiple delivery channels have been used, including community health workers, midwives and traditional birth attendants ${ }^{(17)}$.

Although there are numerous efficacy studies of breastfeeding promotion interventions and some large-scale interventions using project-supported staff ${ }^{(13,18)}$, there are few studies of the effectiveness of programmes operating 
at scale within routine health systems ${ }^{(19,20)}$ where impact may be reduced ${ }^{(1)}$. There is also very little research related to methods of training those delivering breast-feeding promotion interventions ${ }^{(10,21)}$. In the current paper we report the rates of exclusive breast-feeding associated with a breast-feeding counselling intervention delivered within Brazil's primary health-care programme to a population approaching 2 million by community health agents (CHA) who received $20 \mathrm{~h}$ of training directed at breast-feeding counselling and practical skills. We compare these rates with those pre-intervention when CHA received $4 \mathrm{~h}$ of training on breast-feeding with no emphasis on counselling or practical skills. The study thus addresses two research gaps: (i) comparative effectiveness of different training strategies for promotion of exclusive breast-feeding; and (ii) the effectiveness of multifunctional CHA delivering counselling at scale within routine health services.

\section{Methods}

\section{Location}

The study site was the city of Recife, the capital of the State of Pernambuco, north-east Brazil. This municipality has six administrative districts. In 1992 the municipality introduced the Community Health Agents Programme (Programa Agentes Comunitarios de Saúde, or PACS) which focused on maternal and child health. In 1998 the municipality started to replace PACS with the Family Health Programme (Programa Saúde da Família) in which comprehensive health-care services are delivered by teams typically comprising a doctor, a nurse, a nursing assistant and at least four CHA per 3000-4000 inhabitants ${ }^{(22,23)}$. The Programme expanded rapidly after 2003, and by $2007-08$ there were 240 health teams covering approximately $60 \%$ of the city's population of 2 million. As the Family Health Programme is more comprehensive in scope than PACS, community health workers have had to become multifunctional and serve all family members, from infancy to old age. Promotion of breast-feeding has been an integral part of both PACS and the Family Health Programme yet rates of exclusive breast-feeding have been disappointing, and in 1999 the median duration of exclusive breast-feeding in Recife was $7 \mathrm{~d}^{(24)}$. In 2003 the municipality decided to provide CHA with $20 \mathrm{~h}$ of training on breastfeeding counselling and to request additional postnatal home visits in line with our efficacy trial ${ }^{(5)}$. Plans were made from the outset for evaluation of the Recife intervention.

The breast-feeding counselling intervention was implemented on a phased basis between 2003 and 2008 (see Table 1). Groups of twenty-five to thirty CHA were trained together, and to cover one district took approximately 8-10 weeks. The neighbourhoods served by CHA in these districts are primarily low income, crowded, prone to crime, but with water, sanitation and other basic amenities.
Most babies in Brazil are born in hospital and health policies are favourable towards breast-feeding. In Recife, $70 \%$ of births occur in maternities designated as Baby Friendly. Other supportive measures include 6 months' maternity leave for public sector workers, breast milk banks, and a strictly enforced Code of Marketing for milk formula, feeding bottles and pacifiers. There is HIV testing of pregnant women and HIV prevalence is low (about $0 \cdot 4 \%$ of pregnant women).

\section{The intervention}

The basic training for CHA includes $4 \mathrm{~h}$ of didactic teaching on breast-feeding, emphasizing the physiology of lactation and the benefits of breast milk. In contrast, the training intervention for delivering breast-feeding counselling was longer $(20 \mathrm{~h})$ and emphasized counselling skills and practical aspects of the management of breastfeeding. The syllabus was similar to the $18 \mathrm{~h}$ UNICEF/ WHO course for training Baby Friendly Hospitals, but the content specific to delivery room procedures was replaced by discussions about pacifiers and feeding bottles, reasons why babies cry, psychological aspects of breast-feeding, counselling working mothers and adolescent mothers, healthy eating during pregnancy and lactation, and how to enlist support from family members. The teaching style was also less formal and more interactive, with greater use of small-group activities, case studies and personal reflection. Also used were topics from the WHO/UNICEF Breastfeeding Counselling Course, focusing on how to listen to mothers, establish good relationships, build confidence and offer support.

Each CHA received a copy of the book Helping Mothers to Breastfeed $^{(25)}$ and a small illustrated booklet to use during discussions with mothers at home visits. The training was spread over five afternoons and approximately $70 \%$ involved group activities and practice in discussing key topics. CHA training was led by one of the authors (S.B.C.) who is an accredited lactation counsellor and former BFHI assessor, assisted by three university staff (a paediatrician, a social worker and a psychologist). Others with specialist skills assisted in specific sessions, for example to demonstrate milk expression. No posttraining support or supervision of CHA was provided, apart from the very limited supervision from Family Health Programme nurses.

In preparing for the intervention, discussions were held at municipal and district levels, and with the Coordinator of District CHA, and decisions were made jointly. Each CHA is responsible for up to 150 families and they visit each family once per month. In the regular Family Health Programme this routine is unchanged when a baby is born unless s/he is preterm or low birth weight, in which case two visits in the first month are made. To facilitate breast-feeding counselling it was agreed that CHA should aim to visit families three times in the first month, one of which should be within $7 \mathrm{~d}$ of delivery and another in the 
second week. Thus not only was the training content, style and duration different for the intervention, but the number of home visits expected during the infant's first 6 months was increased from six to eight.

\section{Data collection}

To assess impact of the intervention we compared breastfeeding practices pre- and post-intervention in each district through two cross-sectional surveys of a random sample of infants aged 0-5.9 months, the second round taking place approximately 10 months after the district training. Data were collected by six experienced researchers who were university postgraduates and unconnected with the intervention. Information on breast-feeding and use of water, tea, other liquids, solids, pacifiers and bottles in the previous $24 \mathrm{~h}$ were obtained by interviewing mothers at home, by means of a structured questionnaire. WHO definitions were used: infants were recorded as exclusively breast-fed if they received only breast milk (no water, other liquids or solids) and as breast-fed if they received breast milk plus other food or liquid (including other milk). Sociodemographic data were collected and included antenatal care, maternal age, education, literacy, parity, family structure and household possessions.

Mothers' opinions about CHA breast-feeding counselling, their satisfaction in its delivery and suggestions for improving health services were sought by interviewing mothers from Districts I and II ( $n$ 310). The interviews took place in the mothers' homes between March and April 2008. During the same period CHA in these two districts ( $n$ 99) were interviewed regarding their opinions of the intervention training, application of the training and suggestions for overcoming any difficulties regarding their work. Demographic data for all CHA ( $n$ 1449) were obtained at the time of their training. Breast-feeding knowledge pre- and post-training was assessed for all CHA: forty statements were listed pertaining to lactation and breast-feeding, including local beliefs, and the CHA were asked to indicate which statements were correct.

\section{Sample selection}

We calculated that a sample of 200 mother-infant pairs for each of the six districts would have $80 \%$ power to detect a $12 \%$ difference in point prevalence of exclusive breastfeeding at age 6 months at the $5 \%$ significance level (twosided comparison), assuming a pre-intervention point prevalence of $15 \%$. We allowed an increase of $10 \%$ for possible non-responders.

For each round, to meet the required sample size of 200 mother-infant pairs per district, a random sample of 50-75 CHA per district was made and four infants aged 0-5.9 months per CHA were randomly sampled. Infants aged 0-5.9 months were identified from CHA registers which are continuously updated. There were no exclusion criteria. Districts I and II were combined as District I was small.

\section{Statistical analysis}

All recording forms were pre-coded and checked daily for completeness and consistency. Double data entry was verified with Epi-Info version 6•04. Fisher's exact test was used for comparing point prevalences and the MantelHaenszel test was used to test for homogeneity across age groups. The statistical software package SPSS version $8 \cdot 0$ was used. Statistical significance was taken as $P<0 \cdot 05$.

\section{Etbical permission}

The study was conducted according to the guidelines laid down in the Declaration of Helsinki and all procedures involving human subjects were approved by the Ethical Committee of the Instituto de Medicina Integral Professor Fernando Figueira (IMIP). Written informed consent was obtained from all mothers and CHA.

\section{Results}

Collectively, the 1568 CHAs served approximately 7150 infants aged 0-5.9 months at any one point, and 1266 and 1245 of these mother-infant pairs were sampled for rounds one and two, respectively. There were no refusals to participate among the CHA or mothers.

\section{Intervention training}

Table 1 shows the number of CHA and their distribution by district, and the number who received the intervention training. Training was completed by $1449 \mathrm{CHA}(92 \cdot 4 \%)$. Almost all (92\%) were female, 63\% were aged 30-49 years and 25\% were aged 20-29 years. Most (61\%) had completed secondary school, $24 \%$ had completed primary school and $15 \%$ had incomplete primary education. About two-thirds had worked as a CHA for 7 years or more. The mean knowledge score pre-intervention was 7.56 and improved to $9 \cdot 22$ out of a maximum possible score of 10 .

\section{Community bealth agents' opinions of the training and practices}

Table 2 shows that the training was highly rated by $97 \%$ of the sub-sample. Most (65\%) reported putting the training into practice. Most (83\%) reported that they were

Table 1 Number of community health agents (CHA) by district and the proportion who completed $20 \mathrm{~h}$ of training on breast-feeding counselling, Recife, Brazil, 2003-2008

\begin{tabular}{lcrrrrr}
\hline & & \multicolumn{2}{c}{ Total CHA } & & \multicolumn{2}{c}{ CHA trained } \\
\cline { 3 - 4 } \cline { 7 - 8 } Health district & Year of training & \multicolumn{1}{c}{$n$} & \multicolumn{1}{c}{$\%$} & & \multicolumn{1}{c}{$n$} & $\%$ \\
\hline I & 2008 & 84 & $5 \cdot 4$ & & 80 & $95 \cdot 2$ \\
II & 2008 & 325 & $20 \cdot 7$ & & 304 & $93 \cdot 5$ \\
III & 2003 & 282 & $18 \cdot 0$ & & 263 & $93 \cdot 3$ \\
IV & 2005 & 275 & $17 \cdot 5$ & & 268 & $97 \cdot 4$ \\
V & 2003 & 164 & $10 \cdot 5$ & & 156 & $95 \cdot 1$ \\
VI & 2007 & 438 & $27 \cdot 9$ & & 378 & $86 \cdot 3$ \\
Total & & 1568 & $100 \cdot 0$ & & 1449 & $92 \cdot 4$ \\
\hline
\end{tabular}


Table 2 Opinions of community health agents (CHA; $n$ 99) about the breast-feeding counselling intervention, Recife, Brazil, 2008

\begin{tabular}{|c|c|c|c|}
\hline \multirow[b]{2}{*}{ CHA opinions } & \multicolumn{3}{|c|}{ Total } \\
\hline & $n$ & $\%$ & $95 \% \mathrm{Cl}$ \\
\hline Opinion on training (very good/good) & 96 & $97 \cdot 0$ & $91 \cdot 4,99 \cdot 4$ \\
\hline \multicolumn{4}{|l|}{ Training duration } \\
\hline Adequate & 73 & $74 \cdot 7$ & $63 \cdot 9,82 \cdot 1$ \\
\hline Short & 5 & $5 \cdot 1$ & $1 \cdot 7,11 \cdot 4$ \\
\hline Long & 21 & $21 \cdot 2$ & $13 \cdot 6,30 \cdot 6$ \\
\hline Would like refresher & 71 & $71 \cdot 7$ & $61 \cdot 8,80 \cdot 3$ \\
\hline \multicolumn{4}{|l|}{ When } \\
\hline After 6 months & 40 & $40 \cdot 3$ & $28 \cdot 9,52 \cdot 5$ \\
\hline After 1 year & 59 & $59 \cdot 7$ & $47 \cdot 5,71 \cdot 1$ \\
\hline \multicolumn{4}{|l|}{ Have put the training into practice } \\
\hline Yes & 64 & $64 \cdot 6$ & $54 \cdot 4,74 \cdot 0$ \\
\hline More or less & 28 & $28 \cdot 3$ & $19 \cdot 7,38 \cdot 2$ \\
\hline No & 7 & $7 \cdot 1$ & $2 \cdot 9,14 \cdot 0$ \\
\hline Achieved home visit in first week after maternity hospital discharge & 90 & $90 \cdot 9$ & $83 \cdot 4,95 \cdot 8$ \\
\hline Have increased number of breast-feeding visits & 82 & $82 \cdot 7$ & $73 \cdot 7,89 \cdot 6$ \\
\hline \multicolumn{4}{|l|}{ How many visits $0-6$ months? } \\
\hline Not increased & 17 & $17 \cdot 2$ & $10 \cdot 3,26 \cdot 1$ \\
\hline $6-7$ & 15 & $15 \cdot 2$ & $8 \cdot 7,23 \cdot 8$ \\
\hline$\geq 8$ & 67 & $67 \cdot 7$ & $57 \cdot 5,76 \cdot 7$ \\
\hline \multicolumn{4}{|l|}{ Duration of visits (min) } \\
\hline$<20$ & 18 & $18 \cdot 2$ & $11 \cdot 1,27 \cdot 2$ \\
\hline $20-29$ & 28 & $28 \cdot 3$ & $19 \cdot 7,38 \cdot 2$ \\
\hline$\geq 30$ & 53 & $53 \cdot 5$ & $43 \cdot 2,63 \cdot 6$ \\
\hline Presence of another adult during visit & 46 & $46 \cdot 5$ & $36 \cdot 4,56 \cdot 8$ \\
\hline
\end{tabular}

Table 3 Comparability of the distribution of the samples before and after the breast-feeding counselling intervention according to health district; random samples of mother-infant pairs pre- and post-intervention ( $n 1266$ and $n$ 1245, respectively), Recife, Brazil, 2003-2008

\begin{tabular}{|c|c|c|c|c|}
\hline \multirow[b]{3}{*}{ Health district } & \multicolumn{4}{|c|}{ Sample size* } \\
\hline & \multicolumn{2}{|c|}{ Before } & \multicolumn{2}{|c|}{ After } \\
\hline & $n$ & $\%$ & $n$ & $\%$ \\
\hline $\mathrm{I} / \mathrm{II}$ & 313 & $24 \cdot 7$ & 290 & $23 \cdot 3$ \\
\hline III & 201 & $15 \cdot 9$ & 204 & $16 \cdot 4$ \\
\hline IV & 240 & $18 \cdot 9$ & 244 & $19 \cdot 6$ \\
\hline V & 210 & $16 \cdot 7$ & 223 & $17 \cdot 9$ \\
\hline VI & 302 & $23 \cdot 8$ & 284 & $22 \cdot 8$ \\
\hline Total & 1266 & $100 \cdot 0$ & 1245 & $100 \cdot 0$ \\
\hline
\end{tabular}

*Mothers with infants aged 0-5.9 months.

able to increase the number of home visits to at least eight, and $91 \%$ visited within the first week. Most (72\%) considered refresher training to be desirable to keep up to date and review problems encountered in the field.

\section{Comparability of the samples pre- and post-intervention}

Table 3 shows that the number of mother-infant pairs sampled was comparable between the two rounds within a district, and that the distribution of the sample among the districts was maintained pre- and post-intervention. Further comparability checks showed no significant difference in years of maternal schooling or other sociodemographic variables between the pre- and post-intervention samples $(P=0.66)$ nor in pre-intervention exclusive breast-feeding rates among districts sampled between 2003 and 2008 $(P=0 \cdot 12$; data available on request).

\section{Impact on breast-feeding}

Figures 1 and 2 show, respectively, the proportions of infants exclusively breast-fed and at least partially breast-fed by infant age pre- and post-intervention. Postintervention rates of exclusive breast-feeding were significantly higher than pre-intervention by 10-13 percentage points at ages 3-5.9 months, but the difference in any breast-feeding rates was significant only at $4-4.9$ months.

All levels of maternal education benefited. Post-intervention point prevalence of exclusive breast-feeding for infants aged $<4$ months was $63 \%$ and for those aged $<6$ months was $50 \%$. Comparing rates before and after for the total sample aged $<6$ months showed a significant difference in the proportion exclusively breast-fed ( $45 \cdot 6 \% v .50 \cdot 4 \%, P=0 \cdot 02$ ) but there was no significant difference for any breast-feeding $(82 \cdot 8 \% \quad v$. 85.1\%, $P=0 \cdot 13)$. The Mantel-Haenszel stratified analysis 


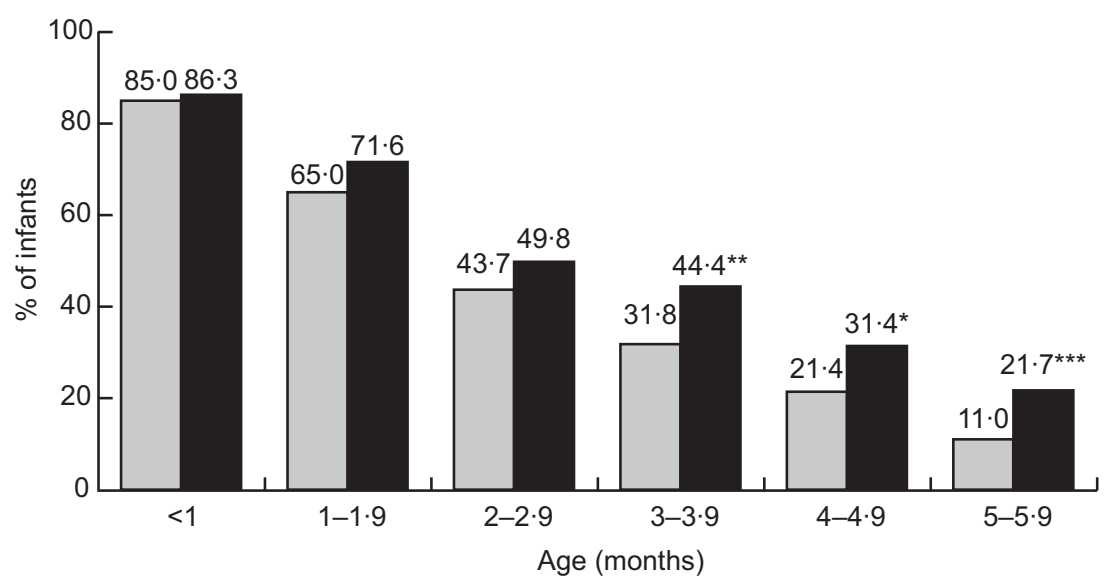

Fig. 1 Proportions of infants exclusively breast-fed from birth to 5.9 months before $(\square)$ and after ( $\square$ ) improved training of community health agents; random samples of mother-infant pairs pre- and post-intervention ( $n 1266$ and $n$ 1245, respectively), Recife, Brazil, 2003-2008. Significant difference, post-intervention $v$. pre-intervention: ${ }^{\star} P=0 \cdot 02,{ }^{\star \star} P=0.01,{ }^{\star \star \star} P=0 \cdot 006$

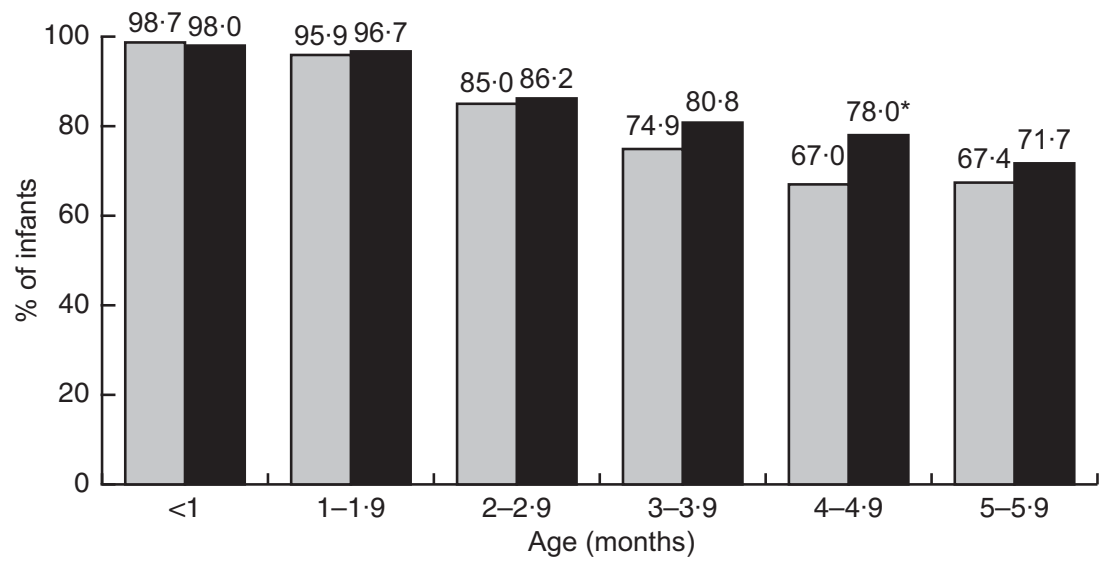

Fig. 2 Proportions of infants at least partially breast-fed from birth to 5.9 months before $(\square)$ and after ( $\square$ ) improved training of community health agents; random samples of mother-infant pairs pre- and post-intervention ( $n 1266$ and $n 1245$, respectively), Recife, Brazil, 2003-2008. Significant difference, post-intervention $v$. pre-intervention: ${ }^{*} P=0 \cdot 01$

showed a significant difference in the last three strata, denoting heterogeneity across the 3-5.9 month age group (Mantel-Haenszel $\chi^{2} P<0 \cdot 001$ ).

\section{Impact on use of water, tea, juice, other milk and pacifiers}

Table 4 shows that the use of water, other milk (with or without cereal) and pacifiers was significantly decreased post-intervention at ages 3-5.9 months. For tea and juice the decrease was significant only at age $2-2 \cdot 9$ and $5-5.9$ months, respectively.

\section{Motbers' opinions}

Most mothers (83\%) reported receiving advice about breast-feeding, and $85 \%$ of these were counselled by CHA. Other sources of advice were also common, especially from other health professionals (74\%). Most mothers (73\%) trusted the CHA's advice and were satisfied with answers given to queries and concerns.

\section{Discussion}

One of the major changes in health provision in Brazil has been the introduction of multifunctional CHA as a totally new cadre of staff and, with more than 250000 CHA now employed nationwide, their training and supervision is a daunting task for health service managers. Thus the setting for the current paper is within a programme that is evolving in an attempt to rectify inherent weaknesses. The breast-feeding counselling intervention was delivered by local staff with existing resources; financial assistance was sought only for the research component to document impact. This contrasts with the LINKAGES Project in Bolivia, Ghana and Madagascar, for example, where additional staff were hired and external technical assistance was provided to improve breast-feeding practices at scale ${ }^{(18)}$.

Since CHA were delivering health care through the municipal health service, the present study measures 


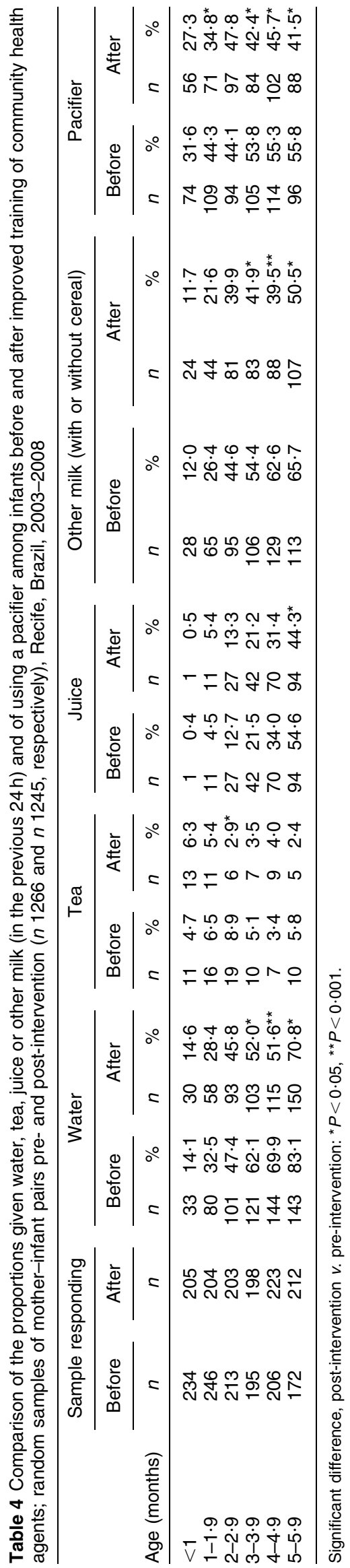

effectiveness. Coverage was good. A methodological limitation is that districts were not randomly allocated to intervention and control groups. The six districts, however, were similar in their pre-intervention exclusive breast-feeding rates and maternal characteristics, and there were no parallel activities that would account for improvements in exclusive breast-feeding rates. Since breast-feeding outcomes were self-reported there is a possible risk of response bias, but we tried to lessen this risk by focusing on infant feeding practices as a whole.

We found improved rates of exclusive breast-feeding when CHA training on breast-feeding was lengthened and directed at counselling and practical skills. Differences in exclusive breast-feeding pre- and post-intervention were statistically significant at ages $3-5.9$ months. Not giving water and not giving other milk were significant factors in achieving exclusivity in this age group. Pacifier use at 3-5.9 months post-intervention decreased by $10-14$ percentage points, which is notable as this behaviour is very resistant to change in Brazil and is associated with shorter breast-feeding duration ${ }^{(26)}$. Pacifier use may be a marker of breast-feeding problems or reduced motivation to breast-feed ${ }^{(27)}$, so a lower usage post-intervention in our study may reflect improved breast-feeding confidence and skill, and a greater desire to breast-feed, as a result of the support and encouragement provided by the CHA.

Assessed cross-sectionally, the proportions of infants who were exclusively breast-fed post-intervention were 86\% (0-1 months), $72 \%$ (1-2 months), $50 \%$ (2-3 months), $44 \%$ (3-4 months), 31\% (4-5 months) and 22\% (5-6 months). These represent a 10-13 percentage point increase above baseline for infants aged 3-5.9 months, which is comparable to the increase found in Bolivia and Ghana in the LINKAGES Project where breast-feeding interventions were delivered at scale through multiple partners, including government health services, nongovernment organizations and radio stations, but lower than the 22 percentage point increase in Madagascar ${ }^{(18)}$. Unlike Madagascar, no attempt was made in Recife to saturate the primary audience with messages, and the number of individuals trained per million of the population was more than three times higher in Madagascar than in Recife. In all three LINKAGE sites, as in our study, great emphasis was given to counselling and negotiation skills and to practical skills in the management of breastfeeding.

Most CHA were able to make three home visits in the first month and to increase the total number to eight during the first 6 months. In a cost-effectiveness analysis Desmond and colleagues ${ }^{(28)}$ modelled six, eight and sixteen postnatal visits and found that although more intensive counselling can be expected to produce better breast-feeding outcomes, there appear to be diminishing marginal returns. When the analysis was applied to KwaZulu-Natal, South Africa, they concluded that the 
model with three antenatal and eight postnatal visits would be the most efficient option. In Recife increasing the number of postnatal visits to eight incurred no additional direct cost to the health service. Increasing the intensity further may improve rates of exclusive breastfeeding, but may not be financially prudent.

Although significant gains were made in rates of exclusive breast-feeding in Recife, a point prevalence rate of $63 \%$ for infants aged $<4$ months and $50 \%$ for infants aged $<6$ months still leaves considerable room for improvement. Additional strategies being considered include extending training to other cadres of health professionals, making greater use of mass media to raise awareness and reinforce messages, and strengthening community involvement in breast-feeding promotion. As a first step the Health Secretariat has initiated joint team discussions (CHA, nurses and doctors) about breast-feeding support so as to integrate service delivery across all cadres of the Family Health Programme. Pre-service training of doctors, nurses and auxiliary health professionals similarly should include breast-feeding counselling.

Brazil was one of the first countries to use television to promote breast-feeding, and television and community radio continue to be used as a free public service during World Breastfeeding Week. Opportunities exist to utilize community radio on a more regular basis. Also, given the wide ownership of cell phones in Brazil, e-messaging is a potential tool for behaviour change that could be considered $^{(29-31)}$. Street theatre led by community members is part of Brazilian culture and CHA utilize this art form in their educational outreach; thus opportunities exist for partnerships between the Family Health Programme and community groups to help establish exclusive breastfeeding as a community norm.

Pre-intervention, many CHA understood the benefits of breast-feeding but their routine training gives no attention to breast-feeding counselling or the management of breast-feeding, and little time is spent in discussion. At the outset we found $\mathrm{CHA}$ to be rather unresponsive to the WHO/UNICEF course format and we quickly changed to a more interactive style in which their knowledge and experiences were utilized and respected. Role plays, discussions and personal reflection were particularly helpful in sensitizing CHA to social and community complexities and in identifying different strategies for solving difficult situations. Having a social worker and psychologist on the training team was useful in this regard.

When discussing the potential contribution of community health workers to child survival, Haines et al. ${ }^{(32)}$ emphasize the need for focused tasks, adequate remuneration, training, supervision and active community involvement. In Brazil, CHA recently obtained job security but there is no national policy for training CHA or for their supervision, which is superficial with little monitoring of key indicators. CHA in the present study desired annual refresher sessions so that they could discuss problems, share experiences and keep up to date. When considering training about breast-feeding, our study reinforces the need to focus on counselling and practical skills, and to use responsive, flexible approaches that draw upon health workers' own knowledge and experiences as these enliven training.

\section{Acknowledgements}

Sources of funding: The study was funded by UNICEF (Recife; grant number 037/2003), the National Council for Research and Development (CNPq; grant numbers 506091/2004-0 and 401986/2005-5) and the Ministry of Health (grant numbers $4641 / 2005$ and 1241/2008), to whom the authors express their thanks. The authors also thank the CNPq for research support to P.I.C.L. (grant number 303377/2010-0) and M.C.L. (grant number 307977/2009-9). Conflicts of interest: The authors have no competing interests. Authors' contributions: All authors helped to conceive the research and design the study, analyse the results and write the paper. S.B.C. trained the CHA. P.I.C.L., M.C.L. and S.H.E. were responsible for data collection and training the research teams. P.G.F. provided logistical support. A.A. prepared the first draft. Acknowledgements: The authors thank the mothers, CHA, managers of the Family Health Programme and the training and research teams for their cooperation.

\section{References}

1. Bhutta ZA, Ahmed T, Black RE et al. (2008) What works? Interventions for maternal and child undernutrition and survival. Lancet 371, 417-440.

2. Jones G, Steketee RW, Black RE et al. (2003) How many child deaths can we prevent this year? Lancet 362, 65-71.

3. Cattaneo A \& Buzzetti R (2001) Effects on rates of breastfeeding of training for the baby friendly hospital initiative. BMJ 323, 1358-1362.

4. Braun MLG, Giugliani ERJ, Soares MEM et al. (2003) Evaluation of the impact of the baby-friendly hospital initiative on rates of breastfeeding. Am J Public Health $\mathbf{9 3}$, 1277-1279.

5. Coutinho SB, Lira PIC, Lima MC et al. (2005) Comparison of the effect of two systems for the promotion of exclusive breastfeeding. Lancet 366, 1094-1100.

6. Leite AJM, Puccini R, Atallah A et al. (1998) Impact on breastfeeding practices promoted by lay counsellors: a randomised and controlled clinical trial. J Clin Epidemiol 51, Suppl. 1, 10S.

7. Morrow AL, Guerrero ML, Shults J et al. (1999) Efficacy of home-based peer counselling to promote exclusive breastfeeding: a randomised controlled trial. Lancet $\mathbf{3 5 3}$, 1226-1231.

8. Haider R, Ashworth A, Kabir I et al. (2000) Effect of community-based peer counsellors on exclusive breastfeeding practices in Dhaka, Bangladesh: a randomised controlled trial. Lancet 356, 1643-1647.

9. Lewin SA, Dick J, Pond P et al. (2005) Lay health workers in primary and community health care. Cochrane Database Syst Rev issue 1, CD004015. 
10. Britton C, McCormick FM, Renfrew MJ et al. (2007) Support for breastfeeding mothers. Cochrane Database Syst Rev issue 1, CD001141.

11. Bland RM, Little KE, Coovadia HM et al. (2008) Intervention to promote exclusive breast-feeding for the first 6 months of life in a high HIV prevalence area. AIDS 22, 883-891.

12. Chapman DJ, Morel K, Anderson AK et al. (2010) Review: breastfeeding peer counseling: from efficacy through scaleup. J Hum Lact 26, 314-326.

13. Tylleskär T, Jackson D, Meda N et al. (2011) Exclusive breastfeeding promotion by peer counsellors in subSaharan Africa (PROMISE-EBF): a cluster-randomised trial. Lancet 378, 420-427.

14. Jolly K, Ingram L, Khan KS et al. (2012) Systematic review of peer support for breastfeeding continuation: metaregression analysis of the effect of setting, intensity and timing. BMJ 344, d8287.

15. Bhandari N, Kabir AKMI \& Salam MA (2008) Mainstreaming nutrition into maternal and child health programmes: scaling up of exclusive breastfeeding. Matern Child Nutr 4, 5-23.

16. The Core Group (2005) 'Scale' and 'Scaling Up'. A CORE Group Background Paper on 'Scaling Up' Maternal, Newborn and Child Health Services, July 11th 2005. Washington, DC: The Core Group; available at http:// www.coregroup.org/storage/documents/Workingpapers/ scaling_up_background_paper_7-13.pdf

17. Bhandari N, Bahl R, Mazumdar S et al. (2003) Effect of community-based promotion of exclusive breastfeeding on diarrhoeal illness and growth: a cluster-randomised controlled trial. Lancet 361, 1418-1423.

18. Quinn VJ, Guyon AB, Schubert JW et al. (2005) Improving breastfeeding practices on a broad scale at the community level: success stories from Africa and Latin America. J Hum Lact 21, 345-354.

19. Casanovas C \& Saadeh R (2009) Scaling up protection, promotion, and support of breastfeeding at the community level. Food Nutr Bull 30, 2 Suppl., S230-S235.
20. Bhutta ZA \& Labbok M (2011) Scaling up breastfeeding in developing countries. Lancet 378, 378-380.

21. World Health Organization (2008) Learning from LargeScale Community-Based Programmes to Improve Breastfeeding Practices. Geneva: WHO.

22. Victora CG, Aquino EML, do Carmo Leal M et al. (2011) Maternal and child health in Brazil: progress and challenges. Lancet 377, 1863-1876.

23. Paim J, Travassos C, Almeida C et al. (2011) The Brazilian health system: history, advances, and challenges. Lancet 377, 1778-1797.

24. Ministry of Health (2001) Prevalência de aleitamento materno nas capitais brasileiras e no Distrito Federal (Breastfeeding Prevalence in Brazilian Capitals and Federal District). Brasília: Ministério da Saúde.

25. Savage King F (1997) Como ajudar as mães a amamentar (Helping Mothers to Breastfeed). Londrina: Universidade Estadual de Londrina.

26. Marques NM, Lira PIC, Lima MC et al. (2001) Breastfeeding and early weaning practices in northeast Brazil: a longitudinal study. Pediatrics 108, e66.

27. Kramer MS, Barr DG, Dagenais S et al. (2001) Pacifier use, early weaning, and cry/fuss behavior. JAMA 286, 322-326.

28. Desmond C, Bland RM, Boyce G et al. (2008) Scaling-up exclusive breastfeeding support programmes: the example of KwaZulu-Natal. PLOS ONE 3, e2454.

29. Fjeldsoe BS, Marshall AL \& Miller YD (2009) Behavior change interventions delivered by mobile telephone shortmessage service. Am J Prev Med 36, 165-173.

30. Krishna S, Boren SA \& Balas EA (2009) Healthcare via cell phones: a systematic review. Telemed $J$ E Health 15, 231-240

31. Cole-Lewis H \& Kershaw T (2010) Text messaging as a tool for behavior change in disease prevention and management. Epidemiol Rev 32, 56-69.

32. Haines A, Sanders D, Lehmann U et al. (2007) Achieving child survival goals: potential contribution of community health workers. Lancet 369, 2121-2131. 